\title{
High-speed Transmission of Information about Car Accident with Communication between Cars
}

\author{
Kosuke Ando ${ }^{\text {a }}$, Yuhki Kitazono ${ }^{\mathrm{a},{ }^{*}}$ \\ ${ }^{a}$ National Institute of Technology, Kitakyushu College, 5-20-1 Shii, Kokuraminami-ku, \\ Kitakyushu-city, Fukuoka 802-0985, Japan \\ *Corresponding Author: kitazono@kct.ac.jp
}

\begin{abstract}
This system, by sharing the data of the vehicle behavior by using the inter-vehicle communication between the surrounding vehicles, perform pre-guess or accident disaster scale guess at the time of the accident vehicle, and the transfer to the surrounding vehicles of their information. By transmitting information at a high speed at the time of the accident to the surrounding vehicles, and it is an object prevent things a second following a disaster.
\end{abstract}

Keywords: Inter-vehicle communication, Accident.

\section{Introduction}

Although the number of incidents of a traffic accident in Japan is decreasing in recent years, it is known that more than a dozen accident by simple calculation in the each prefecture in a day, so we are not necessarily unrelated to traffic accident as shown in Fig. $1^{(1)}$. Moreover, it is known by statistics that the total number of vehicles and density of vehicles increases and in recent years. So it is considered the damage scale increase when accident occurred. When the accident occurred, the accident information transmit to police by the people near by accident site, and then, the information transmit to JARTIC(Japan Road Traffic Information Center), in the end, It is reflected to the Car Navigation System in the conventional. If this is the case, the big overhead occur in pipeline. The people who do not report to the police affect that. Now that the traffic accident occurred, It is most important thing to take measures to the secondary disaster. Also the accident information is a very necessary for the vehicles driving around. Inter-vehicle communication can realize the system that can transmit the information about accident statuses to following vehicles

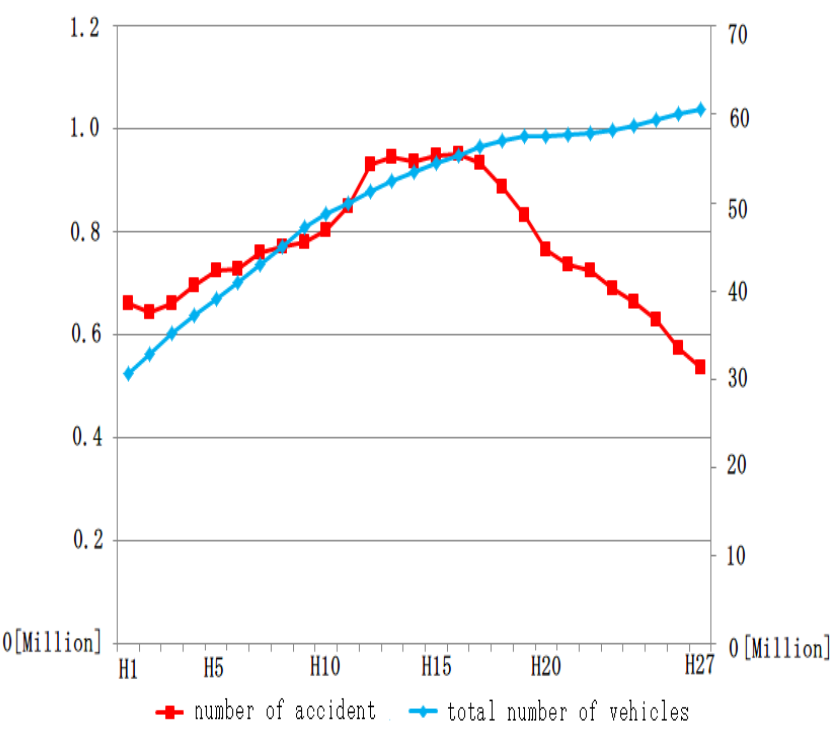

Fig. 1. The graph of number of accident.

rapidly. In this system, It forecast the accident from every information (force interrupt, rate of acceleration, etc....). Its data are shared thorough network between surrounding vehicles. When one vehicle has damaged, surrounding vehicles keep the data about that accident. After an accident occurred, it forecast the scale of the accident and transmits the information to following vehicles.

\section{System}

\subsection{Overview}

In this system, it is assumed that the surrounding vehicles mount same one. This system is mainly composed of Sensor Unit, Processing Unit, and Monitor Unit. In this system, it is assumed that the surrounding vehicles mount same one.

This system is mainly composed of Sensor Unit, 
Processing Unit, and Monitor Unit. The sensor unit detects the behavior of vehicle. It acquires the data (3-Axis Acceleration sensor, GPS module, etc....) from each sensing devices. The processing unit handles the data from sensor unit and detects the dangerous behavior or accident. The monitor unit provides information to driver. The processing unit fetches data from sensors to computer and process the data each pattern. According to pattern, sensor's data transmit to surrounding vehicles with RF modules.

\subsection{Accident Detection Procedure}

In this system, it detects the accident in two steps. First step, detect the accident with sensing devices. As mentioned above, this system detects the dangerous behavior with sensing devices. In this step, it processes the values from sensors (3-Axis Acceleration sensor, Shock sensor). These sensors are used for discrimination of accident in a short interval. If its result shows the accident is clear, the system will start the transmission process immediately. In contrast, GPS module is used in same purpose in a long interval. If accident is not very clear, this system grasps the positions or behaviors of surrounding vehicles by location information from module and discriminate the accident.

Second step, forecast the accident scale with behaviors of surrounding vehicles. After a conclusion the accident, this system estimates the accident scale from suspicious behaviors (extinction of tracking, stop at a suspicious location) of vehicles.

For example, if stream of vehicles stopped at a one intersection that has no traffic lights, the system can conclude that the accident has occurred and the street has closed to traffic at that point.

\subsection{Basic Process}

The action in this system is mainly divided into three patterns. First, the action pattern in normal operation. The normal operation here specifically indicates current traffic status that is a remote possibility. In this pattern, the judgement of step 1 in 2.2 section and transfer the data as shown in Fig. 2(a). Although the data (not including accident information) for following vehicles are basically unnecessary, it is anticipated that the data used for statistics of traffic in future. Second, the action pattern in dangerous behavior. Dangerous behavior indicates the vehicles action that may cause accidents. If vehicles such these behavior approach, it anticipate that each vehicle damaged and share the data to surrounding vehicles for keeping accident status

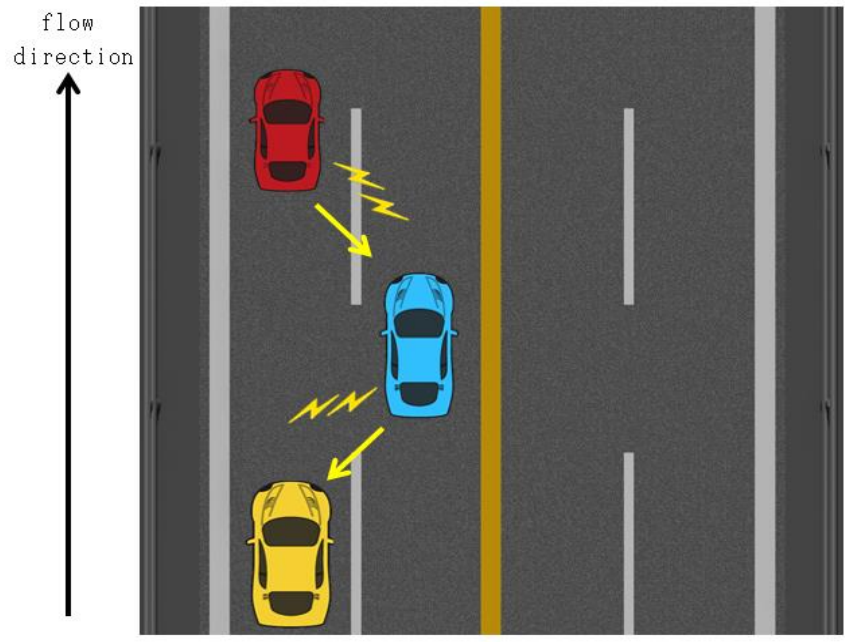

(a) Pattern on normal operation.

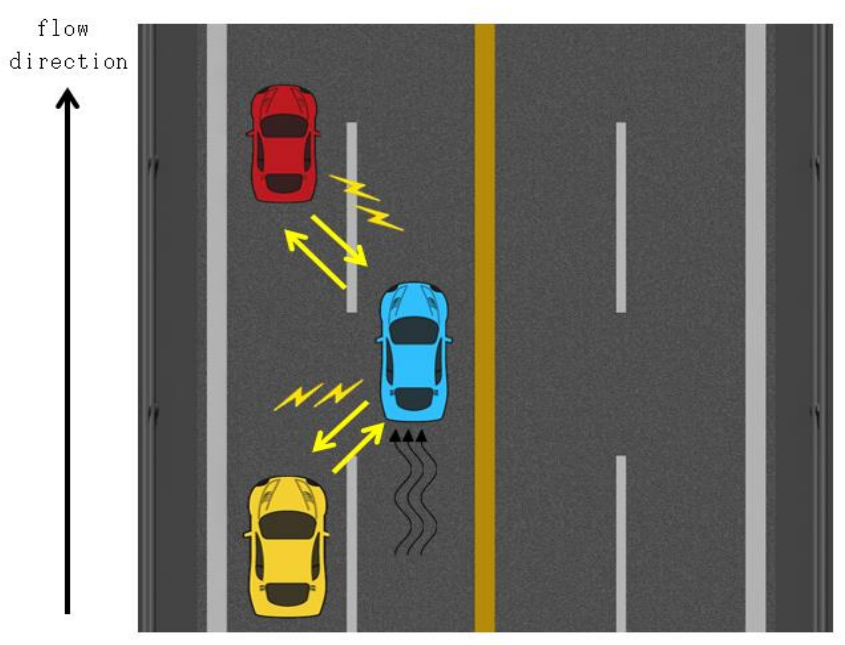

(b) Pattern on dangerous behavior.

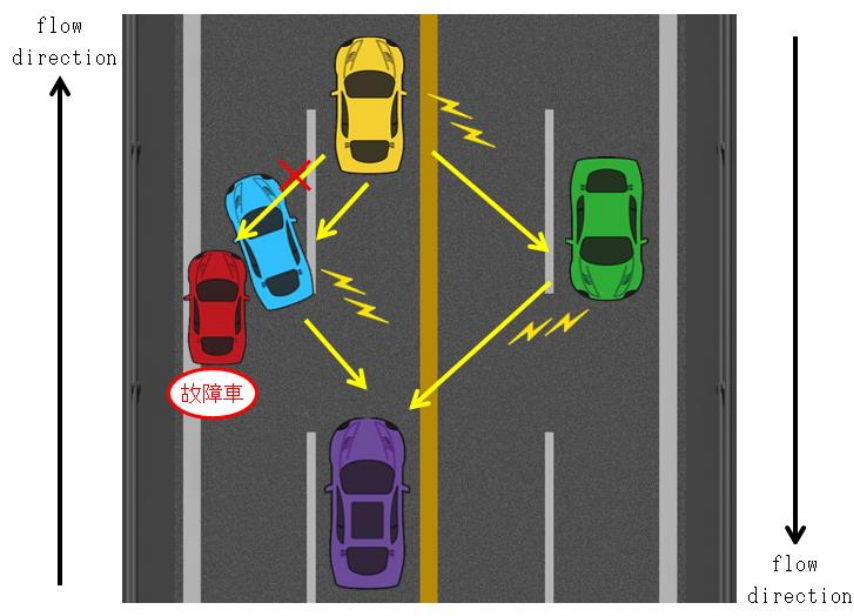

(c) Pattern on accident occurred.

Fig. 2. Pattern. 
as shown in Fig. 2(b). Third, the action pattern in accident occurred. This action operates after a judgement of step 2 in 2.2 section. And also, this operation means it begins to affect caused by the accident. The accident vehicle (or surrounding vehicles) broadcast the information of accident with RF module and hop the data to following vehicle after another as shown in Fig. 2(c).

\subsection{Transmit the Information of Accident}

The information of accident transmits to surrounding vehicles with hopping function on this system. Hopping technology can transfer data at a distance by repeating the flow of transferring the data to the neighboring communication node. By using it, implement a high-speed and long distance communication.

\subsection{Data Division}

When the system has detected the dangerous behavior by sensing devices, the data will transmit immediately. Because it is assumed that the system is damaged by accident. This function is implemented on network imitating RAID (Redundant Arrays of Inexpensive Disks) System. This function will be operating while the vehicles that drive dangerous exist around.

\section{Experiment}

The experiments were conducted to realize this system.

\subsection{Measure the Time from Connection to Disconnection}

In order to realize the high-speed transmission between vehicles, it is most needed to shorten the process time a series of processes from connection until the system would have finished to send the data. Therefore, it would be required to measure how much time it takes, the experiment was conducted. XBee ZigBee module is used as communication module. Although Wi-Fi modules were considered as a communication module in this experiment, it takes more time to establish communication because of the protocol of Wi-Fi has a number of negotiations on the specification. Prepare two Xbee modules and approach slowly from distant position to receivable position. At the moment of catch the signal (catch the RSSI), transmit the data immediately. We have examined the transfer time and integrity of the data each for 10 [Byte], $1 \mathrm{k}$ [Byte] and 10k [Byte]. 10 [Byte] transmitting is assumed the case of
Table 1. The transfer time and consistency of data with $\mathrm{XBee}(38400[\mathrm{bps}])$.

\begin{tabular}{|c|c|c|c|}
\hline $\begin{array}{c}\text { Amount of } \\
\text { data } \\
\text { [Byte] }\end{array}$ & 10 & $1 \mathrm{k}$ & $10 \mathrm{k}$ \\
\hline $\begin{array}{c}\text { Expected } \\
\text { value } \\
\text { [ms] }\end{array}$ & 2.1 & 213 & 2130 \\
\hline $\begin{array}{c}\text { Experiment } \\
\text { value } \\
\text { [ms] }\end{array}$ & 4.1 & 620 & 5800 \\
\hline $\begin{array}{c}\text { Consistency } \\
\text { match }\end{array}$ & match & match \\
\hline
\end{tabular}

emergency. $1 \mathrm{k}$ [Byte] transmitting is assumed the case of transmission data of the accident information per vehicle. 10k [Byte] transmitting is assumed the case of transmission data of the accident information surrounding vehicles. This experimental result is shown in Table 1.

\subsection{Collision Detection with Acceleration Sensor}

In order to detect collision and dangerous behaviors, 3 -axis acceleration sensor is used. Driving speed is derived from the change rate of the position information from GPS.

This system will be able to judge exactly whether it is accident or not by using the both of data. Smartphone (Android Platform) is used for using 3-axis acceleration sensor because of using a lot of hardware element lead to the cost to increase. By using the personal products, become easy to interchange the data. The smartphone can also be used as a display, so use it as a monitor for in this experiment. Attach the USB-OTG cable to the Android device and transfer the data to processing unit (micro controller) with USB-UART converter. We used RX-62G manufactured by Renesas Electronics as a microcontroller. It is equipped CAN (Controller Area Network) function. CAN function frequently is used in communication between the vehicle equipment. For that reason, to use the microcontroller is convenient. This system will transfer the data from microcontroller to Android device if it exceeds a certain threshold. This experimental result is shown in Fig. 3. 


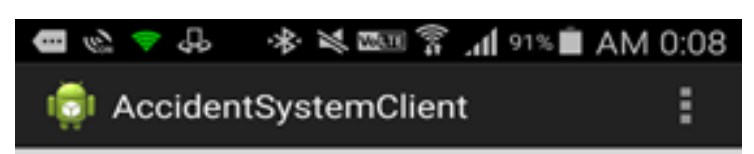

vector $\mathrm{X}:-0.59017074$

vector $Y: 0.9768343$

vector $\mathrm{Z}: \mathbf{9 . 7 3 9 0 1 4}$

Accident has not occured.

(a) Status: Standby.

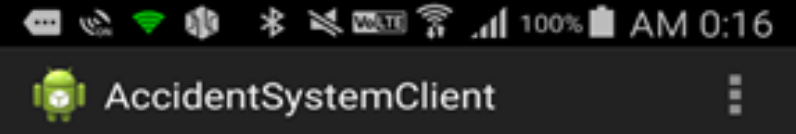

vector X: 8.026934769004583

vector $Y:-0.022744916$

vector $Z: 9.858126$

Accident has occured.

Fig. 3. Collision Detection on Android Monitor.

\section{Conclusions}

From the experiments, it is important to adjust the timing for transferring data in this system. Prevention the false detection of the accident, select the communication path by sensors, and improvement of convenience are future technical issue. About detection performance, we will deal with it by using the sampling-data from sensors and comparing the data from surrounding system. About improvement of convenience, it is assumed that it is important to work with the familiar systems in the same way as this time using a smartphone.

\section{References}

(1) The Metropolitan Police Department, The generation status of the traffic accident in 2015, March, 2018 